\title{
Comparative Evaluation of Microscopy and ELISA in Diagnosis of Cryptosporidiosis in HIV and Non-HIV Patients
}

\author{
A. Tejashree*, P.B. Pooja and K. Sharath Babu \\ Department of Microbiology, JSS Medical College and Hospital, Mysore, India \\ *Corresponding author
}

\section{A B S T R A C T}

\section{Keyw ords \\ Cryptosporidium \\ parvum \\ Immuno- \\ compromised \\ patients.}

Article Info

Accepted:

12 December 2016

Available Online:

10 January 2017
Cryptosporidium is one of the agents associated with diarrhoea in human immune deficiency virus patients. C. parvum is the most common parasite causing diarrhoea in immunocompromised patients. Microscopy is the diagnostic reference standard for the detection of the parasite but it is labour extensive and requires experience. A total 100 stool samples of the subjects attending ICTC and diarrhoea cases admitted in the JSS hospital from March - December 2013 were included in the study 50 subjects were HIV positive and 50 subjects were non HIV (including controls). All the samples were subjected to wet mount, Modified ZN staining and ELISA. Out of 100 samples $9(9 \%)$ were positive by ELISA, 5(5\%) were positive by modified $\mathrm{ZN}$ only one sample was positive by all the 3 methods. Along with cryptosporidium other parasites were also detected like Isospora belli,Giradia, Cysts of Blastocystis, Eggs of Hookworm, Entamoeba histolytica. Based on our data ELISA is an useful assay for the diagnosis cryptosporidium in immunocompromised individuals.

\section{Introduction}

Cryptosporidium species are coccidial protozoan parasites classified as emerging pathogens by the Centre for Disease and Prevention (CDC). These protozoan parasites inhabit the intestinal and respiratory surface of mammals, birds and reptiles and causes human clinical syndrome of cryptosporidiosis (Jayalakshmi et al., 2008; Parisi et al., 1995).

Cryptosporidiosis is a worldwide infection with prevalence rates being higher in developing ( 5 to $10 \%$ ) than in developed (1 to $3 \%$ ) countries. It is more common in young children (ages 1 to 4 years) than in adults, with the exception of patients with AIDS, and occurs more frequently in the warmer months of the year (Rosenblatt, 1993).

Organisms of the genus Cryptosporidium are small $(2-4 \mu \mathrm{m})$ coccidian parasites which have only recently been recognized as important enteric pathogens of humans .Although at least 8 recognised species of Cryptosporidium are considered valid, human cryptosporidiosis is primarily attributed to C.parvum (Michael, 2002).

The common clinical manifestation of Cryptosporidiosis is profuse and watery diahorrea often containing mucus but rarely blood and leucocytes. Other clinical 
symptoms include abdominal cramps, low grade fever, nausea and vomiting. Infection in immune competent host is self limited with duration of approximately 2 weeks. In AIDS patients Cryptosporidiosis generally causes chronic, severe and debilitating voluminous watery diahorrea. It may be accompanied by severe abdominal cramps, weight loss, anorexia, malaise, low grade fever (Hellard et al., 2003).

Cryptosporidium oocysts may be hard to detect except in laboratories with the repeated and continuous exposure to positive specimens that allows diagnostic proficiency. Alternatively, oocyst antigen capture methods such as enzyme immune assays (EIAs) or immunochromatographic-lateral flow (ICLF) assays may be used, andpositive reactions must be confirmed by using a suitable confirmatory test. Due to tiny size of these oocysts, differential staining using the modified Ziehl-Neelsen technique \& wet mount preparation method have limited value for the detection of Cryptosporidium in faecal samples where oocysts can easily be confused with other material present in the samples (Beauty).

Recently, several groups have described the use of enzyme-linked immunosorbent assays (ELISA) for the diagnosis. Enzyme-linked immunosorbent assays (ELISAs) have been reported to be up to 10 times more sensitive than acid-fast staining, making the ELISA method currently the "gold standard" for antigen detection in infected stool samples.

\section{Materials and Methods}

The study was conducted in the department of microbiology at JSS hospital over a period of ten months from March to December 2013. A total of 100 subjects were included in the study which were divided into 4 groups GROUP 1- HIV positive patient with diahorrea - 25 subjects GROUP 2Asymptomatic HIV positive patients - 25 subjects, GROUP 3- HIV negative patient with diahorrea. -25 subjects, GROUP 4Healthy Individuals as controls - 25 subjects. The cases of bacterial diahorrea were excluded. Patients were provided with a clean,wide mouthed container for collection of stool samples with the instruction to transfer them immediately to the microbiology laboratory. All the stool samples were processed for saline and wet mount and examined under the microscope for the presence of protozoal cysts and tropozites. A second smear was made after the concentration with formalin ether method and stained by modified Kinyoun's acid fast stain and examined under oil immersion lens to detect oocysts of Cryptosporidium parvum. Same samples were processed further by ELISA as per manufacturers guidelines (Savyon Diagnostics Ltd. Israel)

\section{Results and Discussion}

Out of 100 stool samples examined Cryptosporidium antigen was detected in 9 $(9 \%)$ by ELISA, in which it was categorised as follows in Group 1 - 05, Group 2 - 01 and in Group 3-03. No antigen was detected in the control group as shown in Table 1.

Cryptosporidium was found in all age group ,the highest number of patients reported during the study belonged to the age group between 21-30 years (30\%) .Table 2

Cryptosporidium were detected in 9 out of 100 stool samples by ELISA, while 1 was detected by wet mount.5 were detected by modified acid fast staining. Out of 9 sample which were positive by ELISA only 5 were positive for microscopy and only one sample was found to be positive by all the three methods. We found detection of cryptosporidial antigen was better with 
ELISA in comparison with other methods (Table 3:) The other parasitolgical profile showed Isopora belli as the second most common pathogen in HIV patients (4) and (2). in non HIV patients. An equal distribution of Cysts Blastocystis hominis was found in both HIV and non-HIV. Eggs of hook worm, Entamoeba histolyica cysts were detected. Cysts of Giardia were also found both in HIV(2) and non HIV patients(4). Coinfection with Cryptospridium and Isospora belli were seen in 10 symptomatic HIV patients (GROUP 1). 5 had coinfection in HIV negative patients with diahorrea (GROUP 3) as shown in Table 4.

Cryptosporidiosis is a common gastrointestinal disease, and it has been recognized worldwide as a common cause of diarrhea. The disease is widespread in many developed and developing countries (Chalmers et al., 2003). According to the World Health Organization, more than 33\% of global deaths are due to parasitic diseases. Intestinal parasitic infections are among the most common infections in the world responsible for mortality and morbidity.

The diagnosis of Cryptosporidiosis is essential in immunocompromised patients, as it may interfere with therapeutical procedures. Because of the difficulty and time-consuming nature of conventional microscopy examination for the detection of Cryptosporidium oocysts, there is a need for a simple and rapid test for the coprodiagnosis of Cryptosporidium infection (Joseph et al., 2001).

In the present study, stool samples from each subject were collected and screened for the presence of Cryptosporidium by wet mount, modified acid fast staining and ELISA.

Our study showed significant number of Cryptosporidium infection in HIV patients
(28\%) than in non HIV patients $(6 \%)$. The study conducted by Jayalakshmi et al., reported $12.4 \%$ in HIV patients. Satheesh kumar et al., \& Singh et al., reported 10.8 to $43 \%$ of Cryptosporidium infection in various parts of the country. Kumar et al. has reported Cryptosporidium as the commonest parasite which was associated with HIV infections in Southern parts of India (Kumar et al., 2002). Our study also projects Cryptosporidium as the most common coccidian parasite which was associated with HIV positive subjects. Studies conducted by Basak et al., and Mohandas et al., have also reported similar results $(2010 ; 2002)$.

The ELISA test is simple and rapid to use and offers a less subjective method than microscopy for detecting this protozoan in faecal samples submitted to a busy diagnostic laboratory. This is a highly sensitive and specific technique, and it is useful for screening large numbers of specimens in a short time period. Also, it does not rely on microscopy skills (Cavasini et al., 2009).

The present study also evaluated different methods of detection of Cryptosporidium in stool samples by microscopy - wet mount, modified acid fast staining, and ELISA. In our study Cryptosporidium was found to be positive in $1(11.1 \%)$ sample by all the 3 methods, 5 samples by ELISA \& modified acid fast staining $(55.5 \%) \& 3$ samples found to be positive only by ELISA (33.3\%). All the positive samples were detected by ELISA $(100 \%)$

Hence detection of Cryptosporidium antigen by ELISA is simple and rapid. Our results show that the ELISA method, in addition to having excellent specificity, exhibits vastly improved sensitivity over those of the routine methods used. ELISA proved to be the most sensitive (100\%) technique indicating the presence of Cryptosporidium. 
Table.1 Total Cryptosporidium Positive among HIV \& non-HIV

\begin{tabular}{|l|c|}
\hline Patient Criteria & Total Positive \\
\hline HIV positive patient with diahorrea & 5 \\
\hline Asymptomatic HIV positive patients. & 1 \\
\hline HIV negative patient with diahorrea. & 3 \\
\hline
\end{tabular}

Table.2 Age Distribution: Association of age with rate of Cryptosporidium infection

\begin{tabular}{|l|l|l|}
\hline \multirow{2}{*}{ Age in years } & \multicolumn{2}{|c|}{$\begin{array}{c}\text { Cryptosporidium in HIV \& non HIV } \\
\text { patients }\end{array}$} \\
\cline { 2 - 3 } & \multicolumn{1}{|c|}{ Negative } & \multicolumn{1}{c|}{ Positive } \\
\hline$\leq 20$ & $7(7.7 \%)$ & $1(11.1 \%)$ \\
\hline $21-30$ & $27(29.7 \%)$ & $1(11.1 \%)$ \\
\hline $31-40$ & $26(28.6 \%)$ & $3(33.3 \%)$ \\
\hline $41-50$ & $18(19.8 \%)$ & $4(44.4 \%)$ \\
\hline $51-60$ & $8(8.8 \%)$ & $0(0.0 \%)$ \\
\hline$>60$ & $5(5.5 \%)$ & $0(0.0 \%)$ \\
\hline Total & $91(100 \%)$ & $9(100 \%)$ \\
\hline
\end{tabular}

Table.3 Comparison of methods of detecting Cryptosporidium in HIV and non-HIV cases

\begin{tabular}{|l|l|}
\hline Methods & $\begin{array}{l}\text { Number } \\
(\mathrm{n}=100)\end{array}$ \\
\hline Wet mount & $01(1 \%)$ \\
\hline Modified ZN staining & $06(6 \%)$ \\
\hline ELISA & $09(9 \%)$ \\
\hline
\end{tabular}

Table.4 Various parasites detected:

\begin{tabular}{|l|c|c|}
\hline \multicolumn{1}{|c|}{ Parasites detected } & HIV & Non-HIV \\
\hline Eggs of Hookworm. & 1 & 2 \\
\hline Giardia. & 2 & 4 \\
\hline Trichuris trichiura. & 0 & 1 \\
\hline Blastocystis Hominis & 1 & 1 \\
\hline Entamoeba histolytica & 0 & 1 \\
\hline Isospora belli & 4 & 2 \\
\hline Cryptosporidium & 6 & 3 \\
\hline
\end{tabular}


Fig.1 Modified ZN Staining Showing Cryptosporidium oocyst

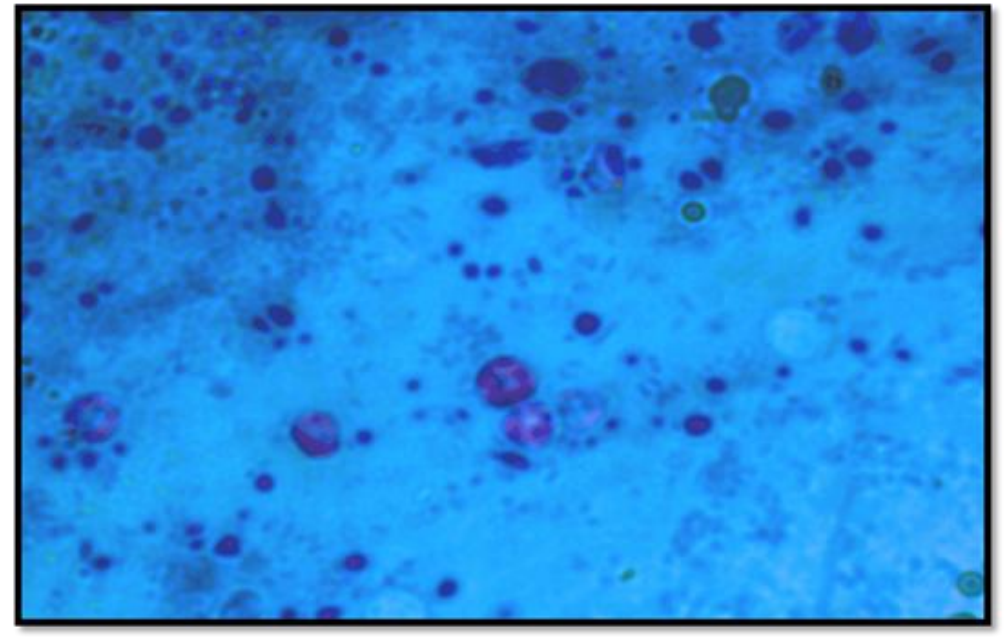

Fig.2 ELISA Test strip for Cryptosporidium Antigen in Stool Samples $\begin{array}{llll}1 & 2 & 3 & 4\end{array}$

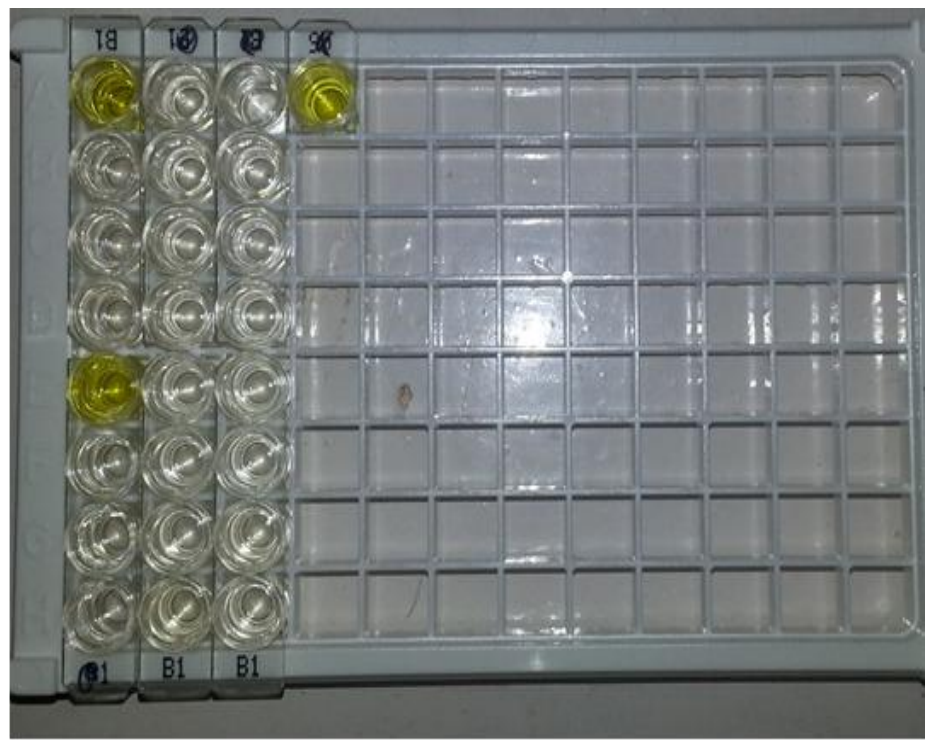

A 1 - POSITIVE CONTROL

B 1 - NEGATIVE CONTROL

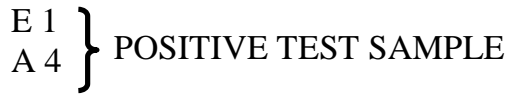


Fig.3 Oocysts of Isosporabelli

Wetmount preparation (40x)

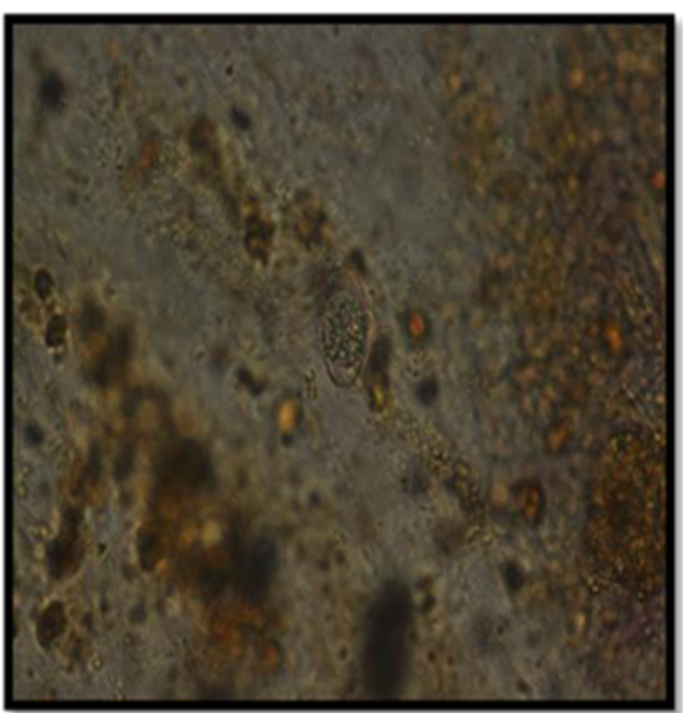

Modified acid fast stain (100x

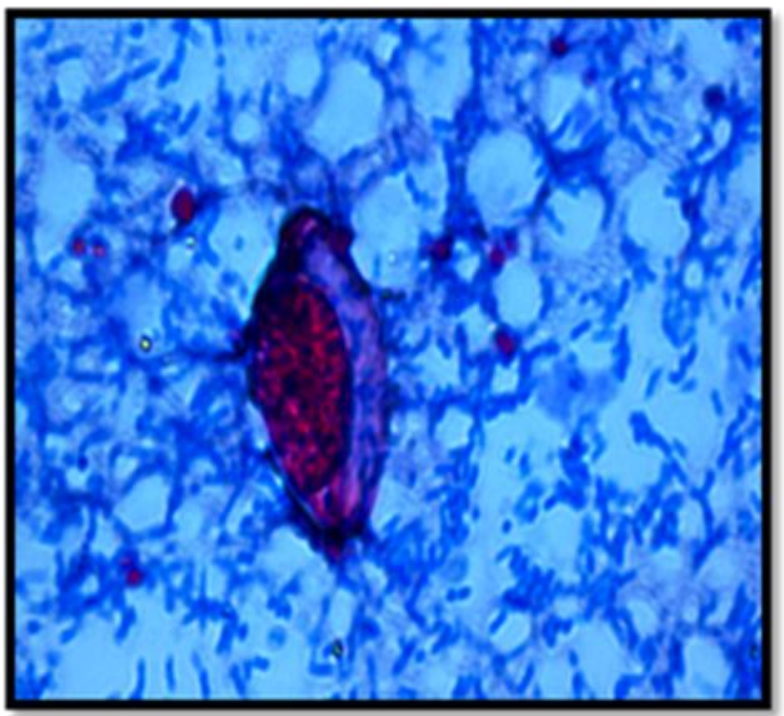

Fig.4 Eggs of Hookworm
Wet mount Preparation (40x):

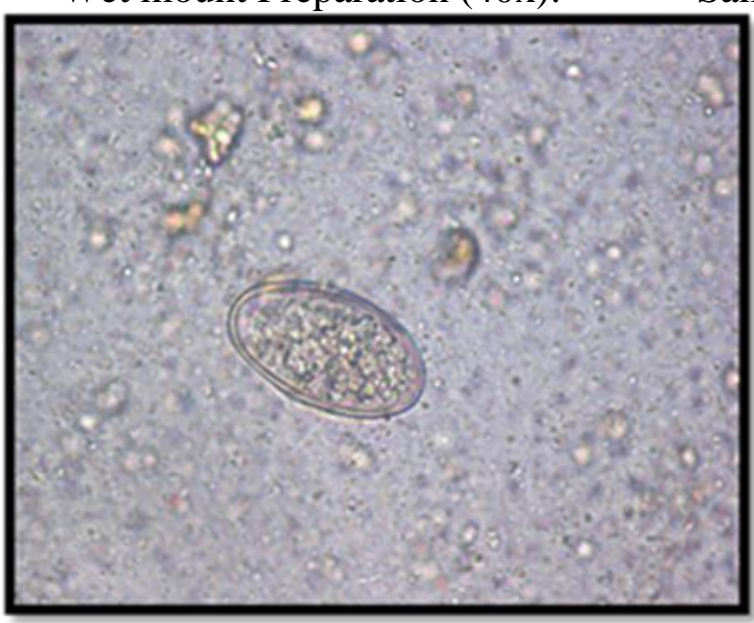

Fabiana Rangel Marques et al conducted a study and reported that, among ninety-four paired samples analyzed the overall sensitivity of the immunoenzymatic assay was $100 \%$, with a specificity of $96 \%$; positive and negative predictive values were $89 \%$ and 100\%, respectively (Cavasini et al., 2009)., Jayalakshmi et al., found ELISA to be a simple, reliable and less subjective test which could be very useful in routine diagnosis and for screening a large number of specimens in short time, particularly in large-scale epidemiological surveys. Barua et al., found Modified acid fast technique to be efficacious method for routine screening of fecal specimens for Cryptosporidium spp in comparison with ELISA (Barua et al., 2008). But in our study ELISA was found to be more efficacious in the detection of Cryptosporidium antigen, as only 05 were detected by modified acid fast staining out of 09 positive which were detected by ELISA. 
Along with Cryptosporidium spp other parasites like Isospora belli, Giradia, Cysts of Blastocystis, Eggs of Hookworm, Entamoeba histolytica were also detected

However ELISA is the most preferred method in the laboratories of the developed countries due to its high specificity and sensitivity, easy usage, fast application and scoring, and easy standardization for determination of Cryptosporidium antigens in stool samples (Hasan, 2008).

In conclusion, cryptosporidiosis is an important water borne disease. The study highlights the importance of evaluation of HIV infected as well as non HIV patients with diarrhoea. For intestinal protozal infection which may help in better management. Based on our data ELISA was found to be more sensitive and specific hence, ELISA is an useful assay for ruling out Cryptosporidiosis in immunocompromised individuals.

\section{References}

Barua, P., Hazarika, N.K., Barua, N., Rasul, E., Laskar, N. 2008. Microscopy for Cryptosporidiosis Screening in Remote Areas. Indian J. Med. Microbiol., 26: 203-204.

Basak, S., Bose, S., Mallick, S.K., Ghosh, A.K. 2010. Intestinal parasitic infections in HIV seropositive patientsA study, J. Clin. Diag. Res., 2433-37.

Beauty, E., Omoruyi, Uchechukwu, U. Nwodo, Chukwuneke, S., Udem, Francis O., Okonkwo. Comparative Diagnostic Techniques for Cryptosporidium Infection; Mol., 19: 2674-2683.

Chalmers, R.M., Casemore, D.P. 2003. Epidemiology and Strain Variation of Cryptosporidium. The Pathogenic Enteric Protozoa: Giardia, Entamoeba,
Cryptosporidium and Cyclospora.,8: 27-42.

Fabiana Rangel Marques, Luciana Ventura Cardoso, Carlos Eugênio Cavasini, Magali Carmem de Almeida, Nair Aparecida Bassi, Margarete Teresa Gottardo de Almeida, Andréa Regina Baptista Rossit1 and Ricardo Luiz Dantas Machado ; Performance of an Immunoenzymatic Assay for Cryptosporidium Diagnosis of Fecal Samples; The Brazilian J. Infectious Dis., 9(1): 3-5.

Hasan Yilmaz, Zeynep Tas-Cengiz, Mutalip Cicek. 2008. Investigation of cryptosporidiosis by enzyme-linked immunosorbent assay and microscopy in children with diarrhea; Saudi Med. J., Vol. 29(4).

Hellard, M., J. Hocking, J. Willis, G. Dore and C. Fairley. 2003. Risk factors leading to Cryptosporidium infection in men who have sex with men. Sex Transm. Infect., 79: 412-414.

Jayalakshmi, J., B. Appalaraju, K. Mahadevan. 2008. Evalution of an Enzyme Linked Immunoassay for the detection of Cryptosporidium antigen in fecal specimens of HIV/AIDS patient. Indian J. pathol. Microbiol., Pp 137138.

Joseph, C.D.C., N.S. Eisenberg, Jeffery, W., Priest, Patrick, J., Lammie, and John, M. Colford, Jr. 2001. The Serologic Response to Cryptosporidium in HIVInfected Persons: Implications for Epidemiologic Research. Centres for Disease Control and Prevention, Atlanta Georgia, 7(6).

Kumar, S.S., Ananthan, S., Lakshmi, P. 2002. Intestinal parasitic infections in HIV infected patients with diarrhoea in Chennai, Indian J. Med. Microbiol., 20(2): 88-89.

Mary, T., Parisi and Philip, M., Tierno, J.R. 1965. Evaluation of New Rapid 
Commercial Enzyme Immunoassay for Detection of Cryptosporidium Oocysts in Untreated Stool Specimens. J. Clin. Microbiol., 33(7): 1963-1965

Michael, J., Arrowood. 2002. In Vitro Cultivation of Cryptosporidium Species. Clin. Microbiol. Rev., 15(3): 390-400.

Mohandas, K., Sehgal, R., Sud, A., Malla, N. 2002. Prevalence of intestinal parasitic pathogens in HIV seropositive individuals in Northern India, Jpn. J. infect. Dis., 55: 83-84.

Newman, R.D., K.L. Jaeger, T. Wuhib, A.A. Lima, R.L. Guerrant and C.L. Sears. Evaluation of an antigen capture enzyme-linked immunosorbent assay for detection of Cryptosporidium oocysts, J. Clin. Microbiol., p. 20802084
Rosenblatt, J.E. \& L.M. sloan. 1993. Evalution of an Enzyme Linked Immunosorbentassay for the detection of Cryptosporidium spp. in stool specimens. J. Clin. Microbiol., pp 14681471.

Satheesh kumar, S., S. Ananthan, P. Lakshmi. 2002. Intestinal Parasitic Infection in HIV Infected Patients with Diarrhea in Chennai. Indian J. Med. Microbiol., 20(2): 88-91.

Singh, A., Bairy, I., Shivananda, P.G. Spectrum of opportunistic infections in AIDS cases. Indian J. Med. Sci., 57(1): 16-21.

World Health Organization. 1991. Basic laboratory methods in medical parasitology, Geneva, 25-26.

\section{How to cite this article:}

Tejashree, A., P.B. Pooja and Sharath Babu, K. 2017. Comparative Evaluation of Microscopy \& ELISA in Diagnosis of Cryptosporidiosis in HIV and Non-HIV Patients. Int.J.Curr.Microbiol.App.Sci. 6(1): 232-239. doi: http://dx.doi.org/10.20546/ijcmas.2017.601.028 